\title{
Potency and penetration of telavancin in staphylococcal biofilms
}

\section{Authors: Kelly R. Kirker, Steve T. Fisher, \& Garth James}

NOTICE: this is the author's version of a work that was accepted for publication in International Journal of Antimicrobial Agents. Changes resulting from the publishing process, such as peer review, editing, corrections, structural formatting, and other quality control mechanisms may not be reflected in this document. Changes may have been made to this work since it was submitted for publication. A definitive version was subsequently published in International Journal of Antimicrobial Agents, 46, 4, October 2015. DOI: http://dx.doi.org/10.1007/s00792-015-0790-x.

Kirker KR, Fisher ST, James GA, "Potency and penetration of telavancin in staphylococcal biofilms," Int J Antimicrob Agents Oct 2015 46(4):451-455.

Made available through Montana State University's ScholarWorks scholarworks.montana.edu 


\section{Potency and penetration of telavancin in staphylococcal biofilms}

Kelly R. Kirker *, Steve T. Fisher, Garth A. James

Center for Biofilm Engineering, Montana State University, Bozeman, MT, USA

\section{Abstract}

Due to the emergence of staphylococcal biofilm infections, the need for advanced antibiotics is cru-cial. The aim of this investigation was to evaluate the potency and penetration of telavancin against staphylococcal biofilms using two different biofilm models. Multiple staphylococcal strains, including meticillin-sensitive Staphylococcus aureus ATCC 29213, vancomycin-intermediate S. aureus ATCC 700787, heterogeneously vancomycin-intermediate S. aureus ATCC 700698 and meticillinsensitive Staphylococ-cus epidermidis ATCC 12228, were grown and treated in dripflow reactors to determine log reductions due to telavancin treatment. After 3 days of biofilm growth and $24 \mathrm{~h}$ of treatment, mean $\log$ reductions for telavancin ranged from 1.65 to 2.17 depending on the bacterial strain tested. Penetration was evaluated qualitatively using confocal scanning laser microscopy to image the infiltration of fluorescently labelled antibiotic into a staphylococcal biofilm grown in a flow cell. Fluorescently labelled telavancin rapidly penetrated the biofilms with no alteration in the biofilm structure.

\section{Introduction}

Multidrug-resistant staphylococcal infections have emerged as a major source both of hospital- and community-acquired infec-tions. Healthcare-associated infections alone cost US hospitals an estimated $\$ 45$ billion dollars annually in 2007 [1]. Many of these infections are associated with bacterial biofilms, which are diffi-cult to eradicate [2]. Biofilm resistance to antibiotics is a result of several mechanisms, which may include failure of the agent to penetrate the full depth of the biofilm, inhibited diffusion within the biofilm, and phenotypic heterogeneity of bacteria within the biofilm [2]. As a result, the need for advanced antibiotics to treat multidrug-resistant staphylococci biofilms is critical.

Telavancin is a semisynthetic, bactericidal lipoglycopeptide. It has a core structure similar to vancomycin but is modified to include a lipophilic side chain [3]. Like vancomycin, telavancin inhibits cell wall synthesis; however, it has a second mechanism of action that disrupts bacterial cell membrane potential and permeability [4]. These two modes of action may explain why telavancin has greater bactericidal activity against staphylococci than van-comycin [4]. The effect of telavancin has been studied in a number of biofilm models. Using a Sorbarod model, telavancin exhibited substantial
* Corresponding author at: Center for Biofilm Engineering, Montana State University, 366 EPS
Building, Bozeman, MT 59717, USA.

Keywords:

Biofilm

Staphylococcal

Telavancin antimicrobial activity against Staphylococcus aureus strains [5]. The Calgary Biofilm Device [6,7], 96-well flat-bottom plates, and biofilms grown on polystyrene disks inside a flow cell [7] have also been used. However, biofilm characteristics, such as cell density and antibiotic tolerance, can vary depending on the biofilm model used [8]. For example, Staphylococcus epidermidis ATCC 12228, used in this study, has been reported to be a non-biofilm forming strain in 96-well plate models using crystal violet assays, yet this strain readily formed biofilms in more robust biofilm culture systems such as flow cells [8]. Furthermore, despite the aforementioned telavancin studies, there are no direct experimental visualisations of the antibiotic penetrating into a biofilm, nor are there studies com-pleted on robust biofilms grown at the solid-liquid-air interface. The aim of this project was to evaluate the potency and penetration of telavancin against staphylococcal biofilms using two different biofilm models. Quantitative analysis of potency was evaluated by plate count to determine log reductions of the antibiotic-treated biofilms relative to control biofilms grown in drip flow reactors (DFRs). Penetration was evaluated qualitatively using confocal scanning laser microscopy to image the penetration of fluorescently labelled telavancin into a biofilm grown in a flow cell.

2. Materials and methods

\subsection{Quantitative analysis of telavancin potency}

A quantitative analysis of antibiotic potency was performed using a Model DFR 110-4 drip flow reactor (BioSurface 


\section{Potency and penetration of telavancin in staphylococcal biofilms}

Kelly R. Kirker *, Steve T. Fisher, Garth A. James

Center for Biofilm Engineering, Montana State University, Bozeman, MT, USA

\section{Abstract}

Due to the emergence of staphylococcal biofilm infections, the need for advanced antibiotics is cru-cial. The aim of this investigation was to evaluate the potency and penetration of telavancin against staphylococcal biofilms using two different biofilm models. Multiple staphylococcal strains, including meticillin-sensitive Staphylococcus aureus ATCC 29213, vancomycin-intermediate S. aureus ATCC 700787, heterogeneously vancomycin-intermediate S. aureus ATCC 700698 and meticillinsensitive Staphylococ-cus epidermidis ATCC 12228, were grown and treated in dripflow reactors to determine log reductions due to telavancin treatment. After 3 days of biofilm growth and $24 \mathrm{~h}$ of treatment, mean $\log$ reductions for telavancin ranged from 1.65 to 2.17 depending on the bacterial strain tested. Penetration was evaluated qualitatively using confocal scanning laser microscopy to image the infiltration of fluorescently labelled antibiotic into a staphylococcal biofilm grown in a flow cell. Fluorescently labelled telavancin rapidly penetrated the biofilms with no alteration in the biofilm structure.

\section{Introduction}

Multidrug-resistant staphylococcal infections have emerged as a major source both of hospital- and community-acquired infec-tions. Healthcare-associated infections alone cost US hospitals an estimated $\$ 45$ billion dollars annually in 2007 [1]. Many of these infections are associated with bacterial biofilms, which are diffi-cult to eradicate [2]. Biofilm resistance to antibiotics is a result of several mechanisms, which may include failure of the agent to penetrate the full depth of the biofilm, inhibited diffusion within the biofilm, and phenotypic heterogeneity of bacteria within the biofilm [2]. As a result, the need for advanced antibiotics to treat multidrug-resistant staphylococci biofilms is critical.

Telavancin is a semisynthetic, bactericidal lipoglycopeptide. It has a core structure similar to vancomycin but is modified to include a lipophilic side chain [3]. Like vancomycin, telavancin inhibits cell wall synthesis; however, it has a second mechanism of action that disrupts bacterial cell membrane potential and permeability [4]. These two modes of action may explain why telavancin has greater bactericidal activity against staphylococci than van-comycin [4].

The effect of telavancin has been studied in a number of biofilm models. Using a Sorbarod model, telavancin exhibited substantial
* Corresponding author at: Center for Biofilm Engineering, Montana State University, 366 EPS
Building, Bozeman, MT 59717, USA.

Keywords:

Biofilm

Staphylococcal

Telavancin antimicrobial activity against Staphylococcus aureus strains [5]. The Calgary Biofilm Device [6,7], 96-well flat-bottom plates, and biofilms grown on polystyrene disks inside a flow cell [7] have also been used. However, biofilm characteristics, such as cell density and antibiotic tolerance, can vary depending on the biofilm model used [8]. For example, Staphylococcus epidermidis ATCC 12228, used in this study, has been reported to be a non-biofilm forming strain in 96-well plate models using crystal violet assays, yet this strain readily formed biofilms in more robust biofilm culture systems such as flow cells [8]. Furthermore, despite the aforementioned telavancin studies, there are no direct experimental visualisations of the antibiotic penetrating into a biofilm, nor are there studies com-pleted on robust biofilms grown at the solid-liquid-air interface. The aim of this project was to evaluate the potency and penetration of telavancin against staphylococcal biofilms using two different biofilm models. Quantitative analysis of potency was evaluated by plate count to determine log reductions of the antibiotic-treated biofilms relative to control biofilms grown in drip flow reactors (DFRs). Penetration was evaluated qualitatively using confocal scanning laser microscopy to image the penetration of fluorescently labelled telavancin into a biofilm grown in a flow cell.

2. Materials and methods

\subsection{Quantitative analysis of telavancin potency}

A quantitative analysis of antibiotic potency was performed using a Model DFR 110-4 drip flow reactor (BioSurface 
Corp., Bozeman, MT) [9,10] equipped with hydroxyapatitecoated glass microscope slides (Clarkston Chromatography, South Williamsport, PA). Antibiotic efficacy was evaluated using singlespecies biofilms of meticillin-susceptible S. aureus ATCC 29213, vancomycin-intermediate S. aureus ATCC 700787, heterogeneously vancomycin-intermediate S. aureus ATCC 700698 and meticillinsusceptible Staphylococcus epidermidis ATCC 12228.

Single-species staphylococcal biofilms were initiated by conditioning the slides with 10\%-strength tryptic soy broth (TSB) (Becton Dickinson \& Co., Sparks, MD) for $10 \mathrm{~min}$ and then inoculating each channel with $1 \mathrm{~mL}$ of an overnight culture $\left(10^{8} \mathrm{CFU} / \mathrm{mL}\right)$ of the test strain. The biofilm was allowed to grow at $37^{\circ} \mathrm{C}$ with $10 \%$ strength TSB medium flowing at a rate of $10 \mathrm{~mL} / \mathrm{h} / \mathrm{channel}$ for a period of 3 days. After the growth period, flow to the reactor was halted, the reactor was set to a horizontal position and $20 \mathrm{~mL}$ of the treatment solution was added to the appropriate channel. The treatment solution consisted of $4 \mathrm{mg} / \mathrm{mL}$ telavancin (Theravance Inc., South San Francisco, CA) in 50\% acidified dimethyl sulfoxide (DMSO), diluted to $80 \mu \mathrm{g} / \mathrm{mL}$ in sterile water. Sterile saline was used as a negative control. Following $24 \mathrm{~h}$ at $37^{\circ} \mathrm{C}$, the treatments were drained and the chambers were rinsed with $20 \mathrm{~mL}$ of additional sterile saline. The slides were removed from the reactor. The biofilms were scraped into $10 \mathrm{~mL}$ of Dey-Engley neutralising broth (Becton Dickinson \& Co.) and were then disaggregated by $30 \mathrm{~s}$ of vortexing, 2 min of sonication and an additional $30 \mathrm{~s}$ of vortexing. The resulting bacterial suspension was then serially diluted 10 -fold with phosphate-buffered saline (PBS) and was plated on tryptic soy agar (Becton Dickinson \& Co.). The plates were incubated at room temperature for 24-48 $\mathrm{h}$ and the number of CFU was counted. Based on the dilution and surface area of the slide, the number of CFU per unit area was calculated and logarithmically (base 10) transformed. The log reduction of each treatment was then calculated relative to the saline-treated control. Each drip flow experiment was repeated four times and the results are presented as the mean \pm standard deviation. Statistical analysis was performed using Minitab v.16 software (Minitab Inc., State College, PA).

\subsection{Fluorescent labelling of telavancin}

Telavancin was labelled with fluorescein isothiocyanate (FITC) (Sigma, St Louis, MO) using standard protein labelling procedures [11]. Telavancin was dissolved in carbonate/bicarbonate buffer at $5 \mu \mathrm{g} / \mathrm{mL}$, mixed with $75 \mu \mathrm{L}$ of FITC solution ( $10 \mathrm{mg} / \mathrm{mL}$ in DMSO) and allowed to react at room temperature for $1 \mathrm{~h}$. The reaction mixture was purified using filtration columns (PD MidiTrap ${ }^{\mathrm{TM}} \mathrm{G}-10$; GE Healthcare Biosciences, Pittsburgh, PA) and was used immediately in the imaging experiments. Concentrations of labelled antibiotics were determined using the relative absorbance at $280 \mathrm{~nm}$ (antibiotic) versus $490 \mathrm{~nm}$ (fluorophore) and the respective molar extinction coefficients. Molar extinction coefficients for the unlabelled antibiotic and the labelling reagent (FITC) were determined by measuring the absorbance versus concentration at $280 \mathrm{~nm}$ (Genesys $^{\mathrm{TM}}$ 10S UV-Vis Spectrophotometer; Thermo Scientific, Waltham, MA).

\subsection{Flow cell imaging}

The penetration of fluorescently labelled telavancin into staphylococcal biofilms was assessed using capillary flow cells [12]. Capillary flow cells (Model FC91; BioSurface Technologies Corp.) were inoculated with $250 \mu \mathrm{L}$ of a $10^{8} \mathrm{CFU} / \mathrm{mL}$ suspension of $S$. aureus ATCC 29213 in TSB. Biofilms were grown for $24 \mathrm{~h}$ with perfusion of $10 \%$-strength TSB medium at a flow rate of $1.0 \mathrm{~mL} / \mathrm{min}$ at $37^{\circ} \mathrm{C}$. Following biofilm growth, the flow cells were mounted on the stage of a Leica SP5 confocal scanning laser microscope
(Leica Microsystems, Inc., Buffalo Grove, IL). Using a 63× water immersion objective, an image plane was selected near the bottom of the biofilm cluster where it was attached to the glass surface, and $108 \mu \mathrm{g} / \mathrm{mL}$ telavancin was introduced into the flow cell at a flow rate of $1.0 \mathrm{~mL} / \mathrm{min}$ at room temperature. Images were simultaneously collected at a wavelength of $500-550 \mathrm{~nm}$ (e.g. green fluorescence, labelled antibiotic) and transmitted light every $3 \mathrm{~s}$ for $12 \mathrm{~min}$. This experiment was repeated three times.

Image analysis was conducted by comparing the mean fluorescence intensity of individual biofilm clusters with the mean fluorescence intensity of the bulk fluid using MetaMorph ${ }^{\circledR}$ 7.7.0.0 image analysis software (Molecular Devices, Downingtown, PA). The resulting data sets resulted in a mean fluorescence intensity versus time for multiple biofilm clusters for both treatments. However, the photomultiplier tube settings were optimised separately for each experimental run, resulting in fluorescence intensity values that could not be directly compared. To normalise these values, the peak mean intensity value for each data set was identified and the remaining data were calculated as a percentage of the peak value. Therefore, the percentage of peak mean fluorescence versus time was determined for multiple biofilm clusters for each labelled antibiotic treatment. The collected images were also used to create time-lapse videos using Imaris x64 7.6.4 software (Bitplane AG, South Windsor, CT) (Supplementary data).

\section{Results}

\subsection{Quantitative analysis of telavancin potency}

After 3 days of biofilm growth and $24 \mathrm{~h}$ of saline treatment, control biofilms had mean log densities of $8.12 \pm 0.40$, $8.12 \pm 0.26,8.08 \pm 0.31$ and $7.93 \pm 0.16 \log _{10} \mathrm{CFU} / \mathrm{cm}^{2}$ for $S$. aureus 29213, S. aureus 700698, S. aureus 700787 and S. epidermidis 12228 , respectively (Fig. 1 ). The respective mean log densities for telavancin-treated biofilms were $5.95 \pm 1.54,6.05 \pm 1.37$, $6.38 \pm 1.39$ and $6.28 \pm 1.38 \log _{10} \mathrm{CFU} / \mathrm{cm}^{2}$ (Fig. 1). The log density for each telavancin group was significantly lower than its control counterpart $(P<0.05)$ using Student's $t$-test assuming one-tail distribution and unequal variances.

Mean log reductions were then calculated by subtracting the mean log density of the treated biofilm from the mean log density of the untreated control. As a result, the mean log reductions for telavancin treatments were $2.17 \pm 1.37,2.07 \pm 1.26,1.70 \pm 1.57$ and $1.65 \pm 1.43 \mathrm{CFU} / \mathrm{cm}^{2}$ for $S$. aureus 29213, S. aureus $700698, S$. aureus 700787 and S. epidermidis 12228, respectively. A general linear analysis of variance (ANOVA) model with a 95\% confidence interval was then fitted to the log reduction data. 'Experiment' was included as a random effect, whilst 'strain' and 'treatment' were included as fixed effects with a potential interaction. Results of this statistical analysis indicated that there was no statistically significant effect of strain $(P=0.943)$ or interaction between strain and treatment $(P=0.871)$. Thus, the effect of telavancin treatment was not strain-dependent in these experiments.

\subsection{Fluorescent labelling of antibiotics}

Molar extinction coefficients determined for telavancin and FITC were determined to be $2808.1 \mathrm{~mol}^{-1} \mathrm{~cm}^{-1}$ and $72,833 \mathrm{~mol}^{-1} \mathrm{~cm}^{-1}$, respectively, and were used to determine the concentration of labelled antibiotic following purification. Optimum labelling was achieved by dissolving $5 \mu \mathrm{g}$ in $1 \mathrm{~mL}$ of PBS and then adding $75 \mu \mathrm{L}$ of a $10 \mathrm{mg} / \mathrm{mL}$ FITC solution. These solutions were prepared fresh for each experiment. 


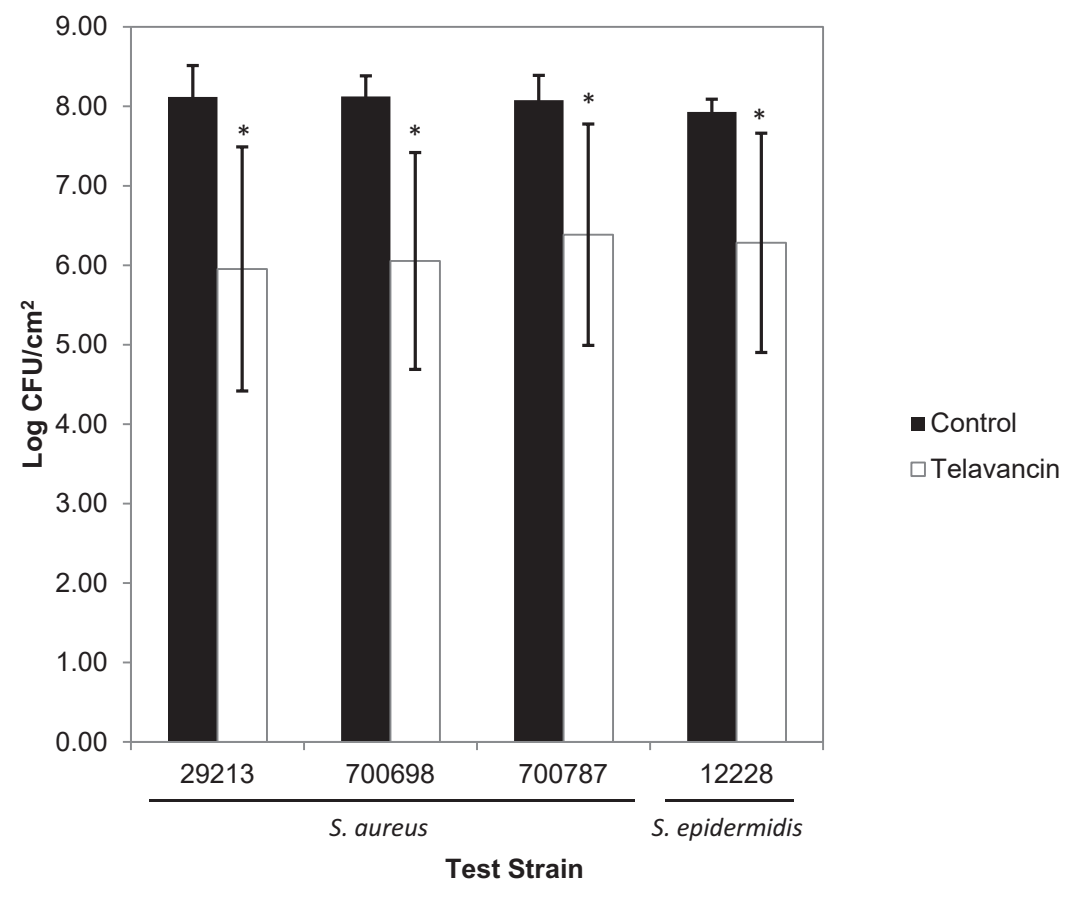

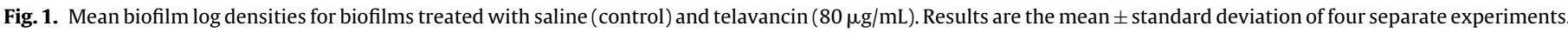
*Significantly different from control $(P<0.05)$.

\subsection{Flow cell imaging}

Staphylococcus aureus 29213 formed extensive, heterogeneous biofilms in the glass capillary flow cell after $24 \mathrm{~h}$ of growth. Thick biofilms formed in the corners of the flow cell and also as large clusters in the middle of the tube walls. These isolated biofilms in the middle of the tube walls were used for the penetration experiments. Time-lapse images were taken of a single focal plane near the bottom of the biofilm cluster where it was attached to the glass surface every $3 \mathrm{~s}$ for $12 \mathrm{~min}$, starting ca. $40 \mathrm{~s}$ prior to introducing the labelled antibiotic. The technique of capturing one image plane within the biofilm rather than an entire $z$-stack of the entire biofilm was selected because preliminary experiments indicated that labelled antibiotic penetration occurred rapidly. The rapid diffusion necessitated a scan rate that was not achievable if imaging the entire biofilm. Therefore, imagining one image plane was selected, using techniques similar to those previously published [12].

Using computer image analysis, a more densely packed region within the biofilm was selected to measure mean fluorescence intensity with time, and an identical region within the flow path was also identified to capture background fluorescence. Fluorescently labelled telavancin rapidly penetrated the biofilm clusters (Fig. 2) with a characteristic S-shaped curve (Fig. 3) [12,13], with the mean maximum fluorescence intensity plateauing at ca. $600 \mathrm{~s}$. No alteration of biofilm structure was observed during the antibiotic exposure.

\section{Discussion}

Telavancin is approved in the USA and Canada for the treatment of adult patients with complicated skin and skin-structure infections due to susceptible Gram-positive pathogens. Telavancin is also approved in the USA and Europe for the treatment of hospital-acquired bacterial pneumonia and ventilator-associated bacterial pneumonia due to $S$. aureus [meticillin-resistant S. aureus (MRSA) only in Europe] when no other alternatives are suitable [14]. Hospital-acquired staphylococcal infections are often associated with bacterial biofilms, which are difficult to eradicate [2]. An often-cited explanation for the failure of antibiotic biofilm treatment is the failure of the antibiotic to penetrate the biofilm [12]. The goal of this project was to visualise telavancin penetration into staphylococcal biofilms and to assess the efficacy of telavancin against robust staphylococcal biofilms.

In the DFR system, biofilms are grown on a solid surface that is continuously wetted with medium at a slow flow rate, providing low shear stress conditions at a solid-liquid-air interface [9]. Although in vitro model systems cannot replicate conditions within infected tissue, the DFR simulates slow flow of body fluids and nutrients that may flow through a biofilm-infected tissue. Furthermore, the substrate material used in this study (hydroxyapatite) is a model material for bone and certain orthopaedic implants. Previous biofilm studies have not examined the effects of telavancin on robust biofilms grown in this manner [5-7]. The robust nature of the DFR biofilm was further demonstrated by the S. epidermidis strain (12228) used in this study. This strain had been reported to be a 'non-biofilm-former' based on simple 96-well plate-based biofilm assays but readily formed biofilms in more complex systems [8] and the study described herein.

In a pharmacokinetic study, the peak plasma concentration for telavancin at the recommended daily therapeutic dosage $(10 \mathrm{mg} / \mathrm{kg} /$ day) was $82.2 \pm 27.3 \mu \mathrm{g} / \mathrm{mL}$ [15]. Therefore, $80 \mu \mathrm{g} / \mathrm{mL}$ telavancin was selected as the DFR treatment concentration because it aligned with the peak plasma concentration using the recommended daily therapeutic dosage. Using this concentration, the mean log reductions for telavancin treatments were $2.16 \pm 1.37,2.07 \pm 1.26,1.07 \pm 1.57$ and $1.65 \pm 1.43 \mathrm{CFU} / \mathrm{cm}^{2}$ for $S$. aureus 29213, S. aureus 700698, S. aureus 700787 and S. epidermidis 12228 , respectively. The variability in these results are quite typical for a treatment using the DFR [10].

Whilst the DFR provided quantitative data for the potency of telavancin, it did not provide for visualisation of the drug penetrating the biofilms. The flow-cell reactor was designed for microscope imaging [12] and allowed for the visualisation of FITC-labelled telavancin penetration into staphylococcal biofilms. 

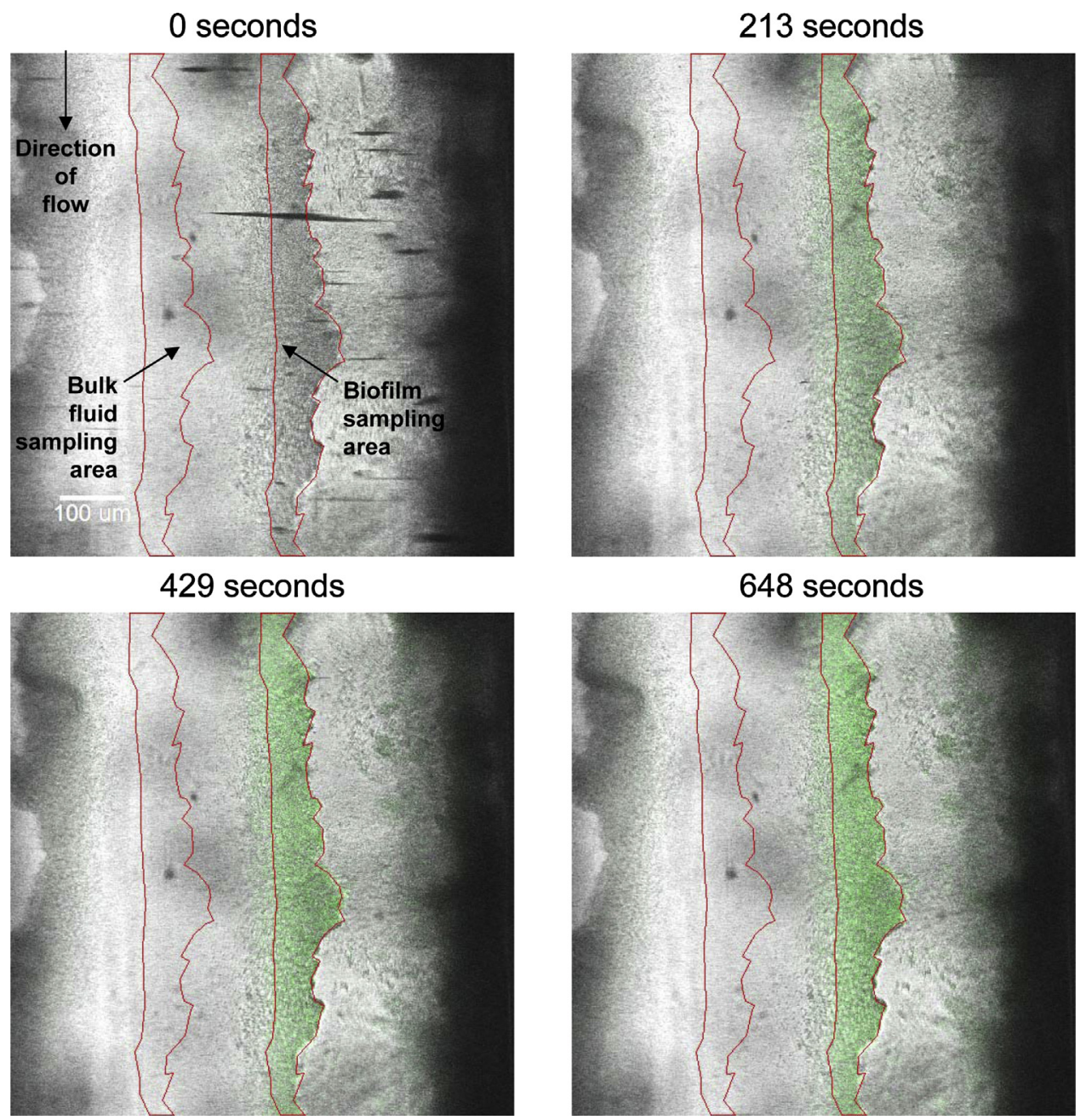

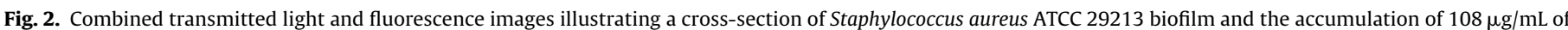

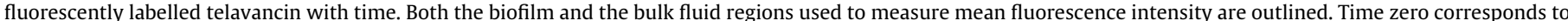
the first appearance of the labelled antibiotic in the focal plane.

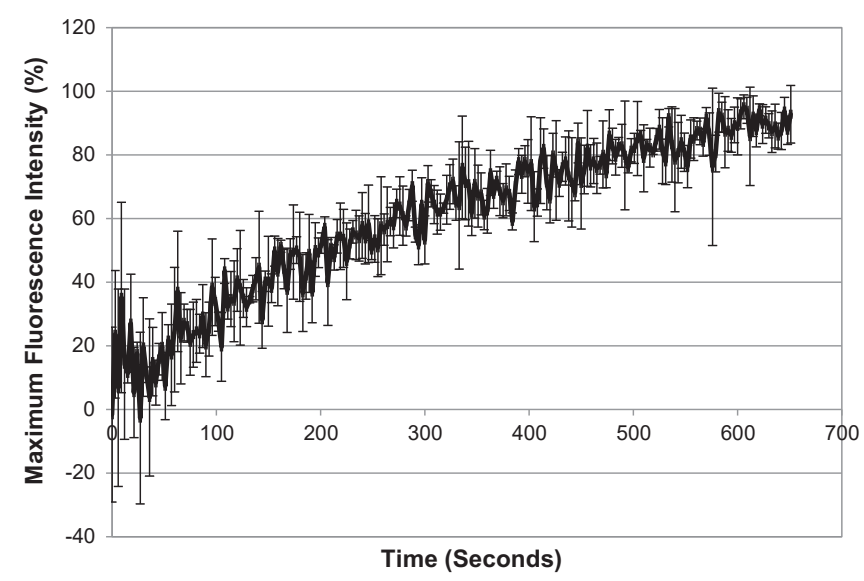

Fig. 3. Image analysis results showing percentage of peak fluorescence intensity versus time for Staphylococcus aureus ATCC 29213 biofilms treated with fluorescently labelled telavancin. Results represent the mean \pm standard deviation of three individual telavancin-treated biofilms. Time zero corresponds to the first appearance of the labelled antibiotic in the focal plane.

However, the telavancin concentration utilised had to be increased compared with the concentration used in the DFR experiments. At $80 \mu \mathrm{g} / \mathrm{mL}$, FITC-labelled telavancin produced a weak fluorescent signal. Increasing the concentration to $108 \mu \mathrm{g} / \mathrm{mL}$ produced a stronger fluorescent signal sufficient for visualisation. This concentration was still within the peak plasma concentration range [15]. The flow-cell experiments demonstrated that telavancin rapidly penetrated the S. aureus 29213 biofilms in a characteristic S-shaped curve (Fig. 3). Since the DFR results (Fig. 1) revealed that telavancin produced similar results regardless of the strain tested, the other staphylococcal strains were not examined in the flow-cell system.

Biofilm resistance to antibiotics is a result of several mechanisms, which may include failure of the agent to penetrate the full depth of the biofilm, inhibited diffusion within the biofilm, and phenotypic heterogeneity of bacteria within the biofilm [2]. The study described herein presents methods to evaluate antibiotic efficacy on robust biofilms and to visualise their penetration into such biofilms. The results indicated that telavancin rapidly penetrated staphylococcal biofilms. Thus, other mechanisms must be involved in the antibiotic tolerance of these biofilms. The techniques described may prove useful in further examining the efficacy of advanced antibiotics designed to treat multidrugresistant biofilms.

Funding: Financial support for this work was provided by Theravance, Inc. (South San Francisco, CA). Telavancin was also supplied by Theravance, Inc.

Competing interests: None declared.

Ethical approval: Not required. 


\section{Appendix A. Supplementary data}

Supplementary data associated with this article can be found, in the online version, at http://dx.doi.org/10.1016/j.ijantimicag.2015. 05.022

\section{References}

[1] Scott II RD. The direct medical costs of healthcare-associated infections in U.S. hospitals and the benefits of prevention. Atlanta, GA: US Centers for Disease Control and Prevention; 2009. p. 1-13.

[2] Costerton JW, Stewart PS, Greenberg EP. Bacterial biofilms: a common cause of persistent infections. Science 1999;284:1318-22.

[3] Leadbetter MR, Adams SM, Bazzini B, Fatheree PR, Karr DE, Krause KM, et al. Hydrophobic vancomycin derivatives with improved ADME properties: discovery of telavancin (TD-6424). J Antibiot (Tokyo) 2004;57:326-36.

[4] Higgins DL, Chang R, Debabov DV, Leung J, Wu T, Krause KM, et al. Telavancin, a multifunctional lipoglycopeptide, disrupts both cell wall synthesis and cell membrane integrity in methicillin-resistant Staphylococcus aureus. Antimicrob Agents Chemother 2005;49:1127-34.

[5] Gander S, Kinnaird A, Finch R. Telavancin: in vitro activity against staphylococci in a biofilm model. J Antimicrob Chemother 2005;56:337-43.

[6] LaPlante KL, Mermel LA. In vitro activities of telavancin and vancomycin against biofilm-producing Staphylococcus aureus, S. epidermidis, and Enterococcus faecalis strains. Antimicrob Agents Chemother 2009;53:3166-9.
[7] Smith K, Gemmell CG, Lang S. Telavancin shows superior activity to vancomycin with multidrug-resistant Staphylococcus aureus in a range of in vitro biofilm models. Eur J Clin Microbiol Infect Dis 2013;32:1327-32.

[8] Dice B, Stoodley P, Buchinsky F, Metha N, Ehrlich GD, Hu FZ. Biofilm formation by ica-positive and ica-negative strains of Staphylococcus epidermidis in vitro. Biofouling 2009;25:367-75.

[9] Goeres DM, Hamilton MA, Beck NA, Buckingham-Meyer K, Hilyard JD, Loetterle LR, et al. A method for growing a biofilm under low shear at the air-liquid interface using the drip flow biofilm reactor. Nat Protoc 2009;4: 783-8.

[10] Buckingham-Meyer K, Goeres DM, Hamilton MA. Comparative evaluation of biofilm disinfectant efficacy tests. J Microbiol Methods 2007;70: 236-44.

[11] Hlady V, Buijs J, Jennissen HP. Methods for studying protein adsorption. Methods Enzymol 1999;309:402-29.

[12] Stewart PS, Davison WM, Steenbergen JN. Daptomycin rapidly penetrates a Staphylococcus epidermidis biofilm. Antimicrob Agents Chemothe 2009;53:3505-7.

[13] Rani SA, Pitts B, Stewart PS. Rapid diffusion of fluorescent tracers into Staphy lococcus epidermidis biofilms visualized by time lapse microscopy. Antimicrob Agents Chemother 2005;49:728-32.

[14] Theravance I. Package insert; 2013. http://www.vibativ.com/ [accessed 01.06.15].

[15] Saraf LJ, Wilson SE. Telavancin, a new lipoglycopeptide antimicrobial, in complicated skin and soft tissue infections. Infect Drug Resist 2011;4: 87-95. 
Corp., Bozeman, MT) [9,10] equipped with hydroxyapatitecoated glass microscope slides (Clarkston Chromatography, South Williamsport, PA). Antibiotic efficacy was evaluated using singlespecies biofilms of meticillin-susceptible S. aureus ATCC 29213, vancomycin-intermediate S. aureus ATCC 700787, heterogeneously vancomycin-intermediate S. aureus ATCC 700698 and meticillinsusceptible Staphylococcus epidermidis ATCC 12228.

Single-species staphylococcal biofilms were initiated by conditioning the slides with 10\%-strength tryptic soy broth (TSB) (Becton Dickinson \& Co., Sparks, MD) for $10 \mathrm{~min}$ and then inoculating each channel with $1 \mathrm{~mL}$ of an overnight culture $\left(10^{8} \mathrm{CFU} / \mathrm{mL}\right)$ of the test strain. The biofilm was allowed to grow at $37^{\circ} \mathrm{C}$ with $10 \%$ strength TSB medium flowing at a rate of $10 \mathrm{~mL} / \mathrm{h} / \mathrm{channel}$ for a period of 3 days. After the growth period, flow to the reactor was halted, the reactor was set to a horizontal position and $20 \mathrm{~mL}$ of the treatment solution was added to the appropriate channel. The treatment solution consisted of $4 \mathrm{mg} / \mathrm{mL}$ telavancin (Theravance Inc., South San Francisco, CA) in 50\% acidified dimethyl sulfoxide (DMSO), diluted to $80 \mu \mathrm{g} / \mathrm{mL}$ in sterile water. Sterile saline was used as a negative control. Following $24 \mathrm{~h}$ at $37^{\circ} \mathrm{C}$, the treatments were drained and the chambers were rinsed with $20 \mathrm{~mL}$ of additional sterile saline. The slides were removed from the reactor. The biofilms were scraped into $10 \mathrm{~mL}$ of Dey-Engley neutralising broth (Becton Dickinson \& Co.) and were then disaggregated by $30 \mathrm{~s}$ of vortexing, 2 min of sonication and an additional $30 \mathrm{~s}$ of vortexing. The resulting bacterial suspension was then serially diluted 10 -fold with phosphate-buffered saline (PBS) and was plated on tryptic soy agar (Becton Dickinson \& Co.). The plates were incubated at room temperature for 24-48 $\mathrm{h}$ and the number of CFU was counted. Based on the dilution and surface area of the slide, the number of CFU per unit area was calculated and logarithmically (base 10) transformed. The log reduction of each treatment was then calculated relative to the saline-treated control. Each drip flow experiment was repeated four times and the results are presented as the mean \pm standard deviation. Statistical analysis was performed using Minitab v.16 software (Minitab Inc., State College, PA).

\subsection{Fluorescent labelling of telavancin}

Telavancin was labelled with fluorescein isothiocyanate (FITC) (Sigma, St Louis, MO) using standard protein labelling procedures [11]. Telavancin was dissolved in carbonate/bicarbonate buffer at $5 \mu \mathrm{g} / \mathrm{mL}$, mixed with $75 \mu \mathrm{L}$ of FITC solution ( $10 \mathrm{mg} / \mathrm{mL}$ in DMSO) and allowed to react at room temperature for $1 \mathrm{~h}$. The reaction mixture was purified using filtration columns (PD MidiTrap ${ }^{\mathrm{TM}} \mathrm{G}-10$; GE Healthcare Biosciences, Pittsburgh, PA) and was used immediately in the imaging experiments. Concentrations of labelled antibiotics were determined using the relative absorbance at $280 \mathrm{~nm}$ (antibiotic) versus $490 \mathrm{~nm}$ (fluorophore) and the respective molar extinction coefficients. Molar extinction coefficients for the unlabelled antibiotic and the labelling reagent (FITC) were determined by measuring the absorbance versus concentration at $280 \mathrm{~nm}$ (Genesys $^{\mathrm{TM}}$ 10S UV-Vis Spectrophotometer; Thermo Scientific, Waltham, MA).

\subsection{Flow cell imaging}

The penetration of fluorescently labelled telavancin into staphylococcal biofilms was assessed using capillary flow cells [12]. Capillary flow cells (Model FC91; BioSurface Technologies Corp.) were inoculated with $250 \mu \mathrm{L}$ of a $10^{8} \mathrm{CFU} / \mathrm{mL}$ suspension of $S$. aureus ATCC 29213 in TSB. Biofilms were grown for $24 \mathrm{~h}$ with perfusion of $10 \%$-strength TSB medium at a flow rate of $1.0 \mathrm{~mL} / \mathrm{min}$ at $37^{\circ} \mathrm{C}$. Following biofilm growth, the flow cells were mounted on the stage of a Leica SP5 confocal scanning laser microscope
(Leica Microsystems, Inc., Buffalo Grove, IL). Using a 63× water immersion objective, an image plane was selected near the bottom of the biofilm cluster where it was attached to the glass surface, and $108 \mu \mathrm{g} / \mathrm{mL}$ telavancin was introduced into the flow cell at a flow rate of $1.0 \mathrm{~mL} / \mathrm{min}$ at room temperature. Images were simultaneously collected at a wavelength of $500-550 \mathrm{~nm}$ (e.g. green fluorescence, labelled antibiotic) and transmitted light every $3 \mathrm{~s}$ for $12 \mathrm{~min}$. This experiment was repeated three times.

Image analysis was conducted by comparing the mean fluorescence intensity of individual biofilm clusters with the mean fluorescence intensity of the bulk fluid using MetaMorph ${ }^{\circledR}$ 7.7.0.0 image analysis software (Molecular Devices, Downingtown, PA). The resulting data sets resulted in a mean fluorescence intensity versus time for multiple biofilm clusters for both treatments. However, the photomultiplier tube settings were optimised separately for each experimental run, resulting in fluorescence intensity values that could not be directly compared. To normalise these values, the peak mean intensity value for each data set was identified and the remaining data were calculated as a percentage of the peak value. Therefore, the percentage of peak mean fluorescence versus time was determined for multiple biofilm clusters for each labelled antibiotic treatment. The collected images were also used to create time-lapse videos using Imaris x64 7.6.4 software (Bitplane AG, South Windsor, CT) (Supplementary data).

\section{Results}

\subsection{Quantitative analysis of telavancin potency}

After 3 days of biofilm growth and $24 \mathrm{~h}$ of saline treatment, control biofilms had mean log densities of $8.12 \pm 0.40$, $8.12 \pm 0.26,8.08 \pm 0.31$ and $7.93 \pm 0.16 \log _{10} \mathrm{CFU} / \mathrm{cm}^{2}$ for $S$. aureus 29213, S. aureus 700698, S. aureus 700787 and S. epidermidis 12228 , respectively (Fig. 1 ). The respective mean log densities for telavancin-treated biofilms were $5.95 \pm 1.54,6.05 \pm 1.37$, $6.38 \pm 1.39$ and $6.28 \pm 1.38 \log _{10} \mathrm{CFU} / \mathrm{cm}^{2}$ (Fig. 1). The log density for each telavancin group was significantly lower than its control counterpart $(P<0.05)$ using Student's $t$-test assuming one-tail distribution and unequal variances.

Mean log reductions were then calculated by subtracting the mean log density of the treated biofilm from the mean log density of the untreated control. As a result, the mean log reductions for telavancin treatments were $2.17 \pm 1.37,2.07 \pm 1.26,1.70 \pm 1.57$ and $1.65 \pm 1.43 \mathrm{CFU} / \mathrm{cm}^{2}$ for $S$. aureus 29213, S. aureus $700698, S$. aureus 700787 and S. epidermidis 12228, respectively. A general linear analysis of variance (ANOVA) model with a 95\% confidence interval was then fitted to the log reduction data. 'Experiment' was included as a random effect, whilst 'strain' and 'treatment' were included as fixed effects with a potential interaction. Results of this statistical analysis indicated that there was no statistically significant effect of strain $(P=0.943)$ or interaction between strain and treatment $(P=0.871)$. Thus, the effect of telavancin treatment was not strain-dependent in these experiments.

\subsection{Fluorescent labelling of antibiotics}

Molar extinction coefficients determined for telavancin and FITC were determined to be $2808.1 \mathrm{~mol}^{-1} \mathrm{~cm}^{-1}$ and $72,833 \mathrm{~mol}^{-1} \mathrm{~cm}^{-1}$, respectively, and were used to determine the concentration of labelled antibiotic following purification. Optimum labelling was achieved by dissolving $5 \mu \mathrm{g}$ in $1 \mathrm{~mL}$ of PBS and then adding $75 \mu \mathrm{L}$ of a $10 \mathrm{mg} / \mathrm{mL}$ FITC solution. These solutions were prepared fresh for each experiment. 


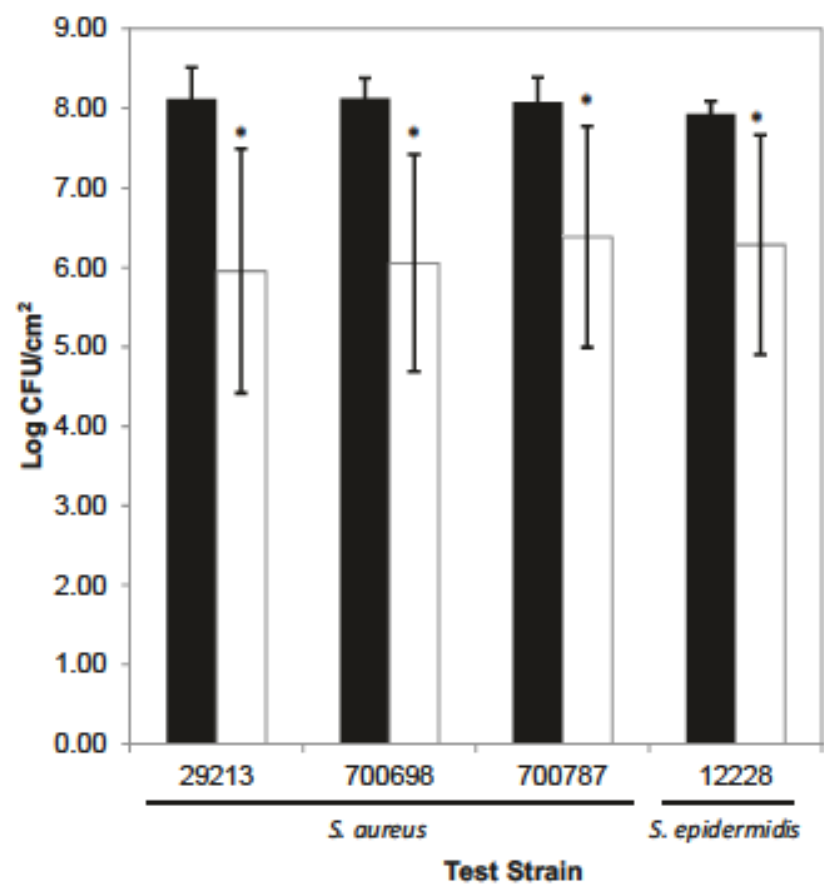

- Control

-Telavancin

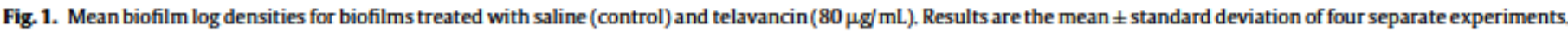
-Significantly different from control $(P<0.05)$.

\subsection{Flow cell imaging}

Staphylococcus aureus 29213 formed extensive, heterogeneous biofilms in the glass capillary flow cell after $24 \mathrm{~h}$ of growth. Thick biofilms formed in the corners of the flow cell and also as large clusters in the middle of the tube walls. These isolated biofilms in the middle of the tube walls were used for the penetration experiments. Time-lapse images were taken of a single focal plane near the bottom of the biofilm cluster where it was attached to the glass surface every $3 \mathrm{~s}$ for $12 \mathrm{~min}$, starting ca. 40 s prior to introducing the labelled antibiotic. The technique of capturing one image plane within the biofilm rather than an entire $z$-stack of the entire biofilm was selected because preliminary experiments indicated that labelled antibiotic penetration occurred rapidly. The rapid diffusion necessitated a scan rate that was not achievable if imaging the entire biofilm. Therefore, imagining one image plane was selected, using techniques similar to those previously published [12].

Using computer image analysis, a more densely packed region within the biofilm was selected to measure mean fluorescence intensity with time, and an identical region within the flow path was also identified to capture background fluorescence. Fluorescently labelled telavancin rapidly penetrated the biofilm clusters (Fig. 2) with a characteristic S-shaped curve (Fig. 3) [12,13], with the mean maximum fluorescence intensity plateauing at ca. $600 \mathrm{~s}$. No alteration of biofilm structure was observed during the antibiotic exposure.

\section{Discussion}

Telavancin is approved in the USA and Canada for the treatment of adult patients with complicated skin and skin-structure infections due to susceptible Gram-positive pathogens. Telavancin is also approved in the USA and Europe for the treatment of hospital-acquired bacterial pneumonia and ventilator-associated bacterial pneumonia due to $S$. aureus [meticillin-resistant $S$. aureus (MRSA) only in Europe] when no other alternatives are suitable [14]. Hospital-acquired staphylococcal infections are often associated with bacterial biofilms, which are difficult to eradicate [2]. An often-cited explanation for the failure of antibiotic biofilm treatment is the failure of the antibiotic to penetrate the biofilm [12]. The goal of this project was to visualise telavancin penetration into staphylococcal biofilms and to assess the efficacy of telavancin against robust staphylococcal biofilms.

In the DFR system, biofilms are grown on a solid surface that is continuously wetted with medium at a slow flow rate, providing low shear stress conditions at a solid-liquid-air interface [9]. Although in vitro model systems cannot replicate conditions within infected tissue, the DFR simulates slow flow of body fluids and nutrients that may flow through a biofilm-infected tissue. Furthermore, the substrate material used in this study (hydroxyapatite) is a model material for bone and certain orthopaedic implants. Previous biofilm studies have not examined the effects of telavancin on robust biofilms grown in this manner [5-7]. The robust nature of the DFR biofilm was further demonstrated by the $S$. epidermidis strain (12228) used in this study. This strain had been reported to be a 'non-biofilm-former' based on simple 96-well plate-based biofilm assays but readily formed biofilms in more complex systems [8] and the study described herein.

In a pharmacokinetic study, the peak plasma concentration for telavancin at the recommended daily therapeutic dosage $(10 \mathrm{mg} / \mathrm{kg} /$ day) was $82.2 \pm 27.3 \mu \mathrm{g} / \mathrm{mL}$ [15]. Therefore, $80 \mu \mathrm{g} / \mathrm{mL}$ telavancin was selected as the DFR treatment concentration because it aligned with the peak plasma concentration using the recommended daily therapeutic dosage. Using this concentration, the mean log reductions for telavancin treatments were $2.16 \pm 1.37,2.07 \pm 1.26,1.07 \pm 1.57$ and $1.65 \pm 1.43 \mathrm{CFU} / \mathrm{cm}^{2}$ for $S$. aureus 29213, S. aureus 700698, S. aureus 700787 and S. epidermidis 12228 , respectively. The variability in these results are quite typical for a treatment using the DFR [10].

Whilst the DFR provided quantitative data for the potency of telavancin, it did not provide for visualisation of the drug penetrating the biofilms. The flow-cell reactor was designed for microscope imaging [12] and allowed for the visualisation of FITC-labelled telavancin penetration into staphylococcal biofilms. 


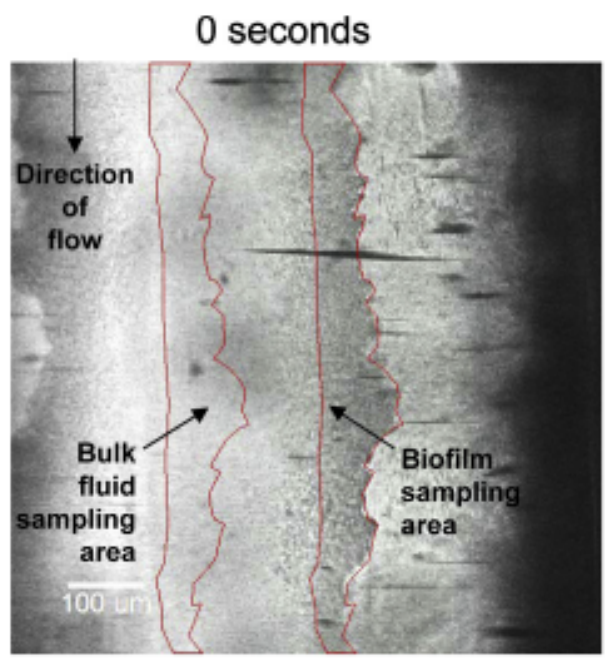

429 seconds

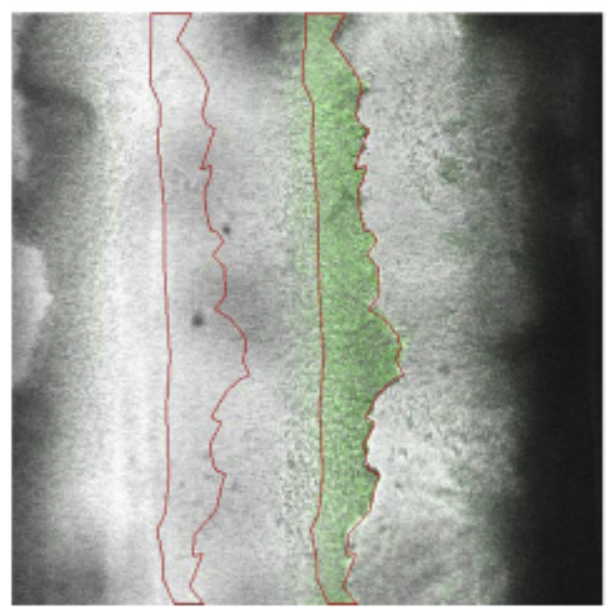

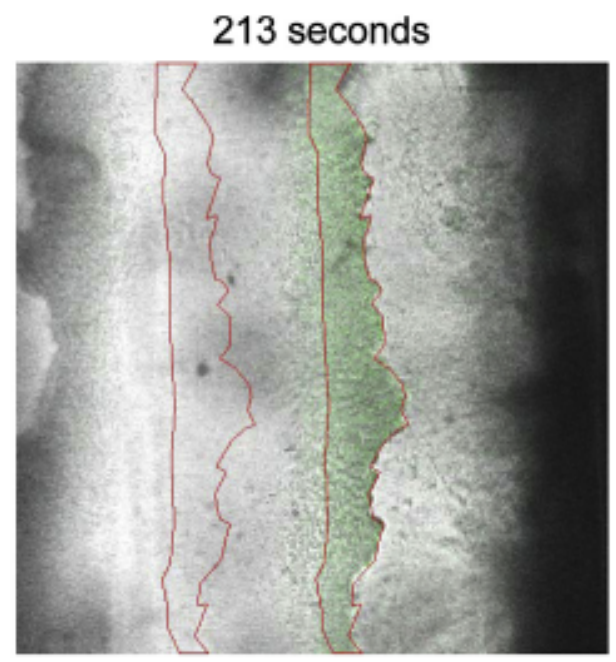

648 seconds

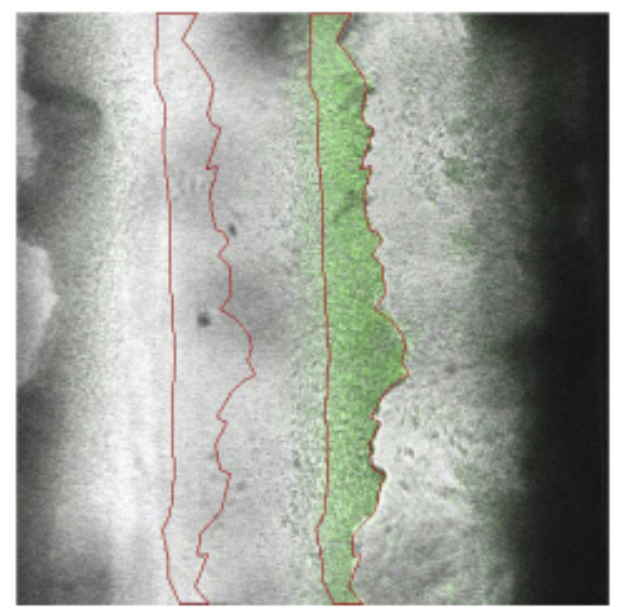

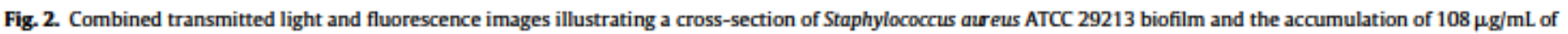

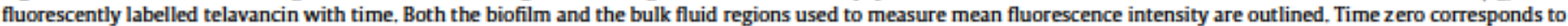
the first appearance of the labelled antibiotic in the focal plane.

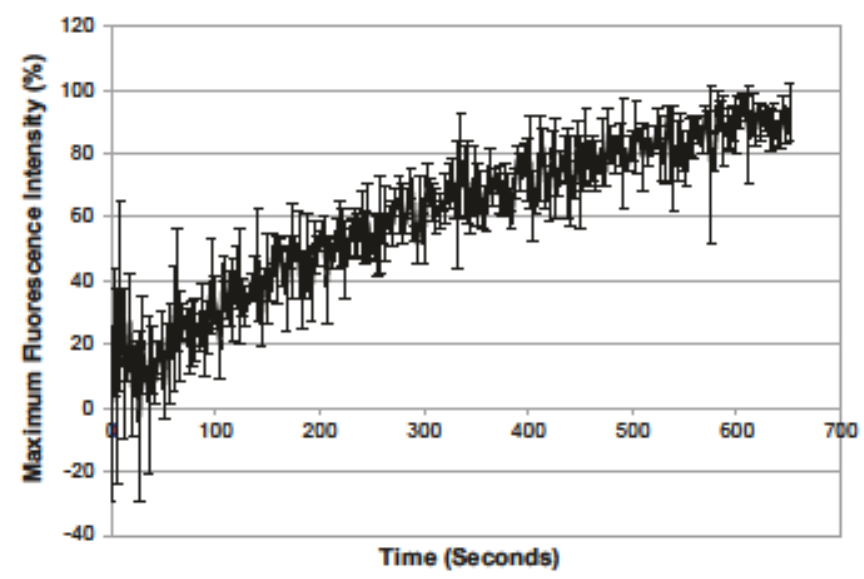

Fig. 3. Image analysis results showing percentage of peak fluorescence intensity versus time for Staphylococcus aureus ATCC 29213 biofilms treated with fluorescently labelled telavancin. Results represent the mean \pm standard deviation of three individual telavancin-treated biofilms. Time zero corresponds to the first appearance of the labelled antibiotic in the focal plane.

However, the telavancin concentration utilised had to be increased compared with the concentration used in the DFR experiments. At $80 \mu \mathrm{g} / \mathrm{mL}$, FITC-labelled telavancin produced a weak fluorescent signal. Increasing the concentration to $108 \mu \mathrm{g} / \mathrm{mL}$ produced a stronger fluorescent signal sufficient for visualisation. This concentration was still within the peak plasma concentration range [15]. The flow-cell experiments demonstrated that telavancin rapidly penetrated the S. aureus 29213 biofilms in a characteristic S-shaped curve (Fig. 3). Since the DFR results (Fig. 1) revealed that telavancin produced similar results regardless of the strain tested, the other staphylococcal strains were not examined in the flow-cell system.

Biofilm resistance to antibiotics is a result of several mechanisms, which may include failure of the agent to penetrate the full depth of the biofilm, inhibited diffusion within the biofilm, and phenotypic heterogeneity of bacteria within the biofilm [2]. The study described herein presents methods to evaluate antibiotic efficacy on robust biofilms and to visualise their penetration into such biofilms. The results indicated that telavancin rapidly penetrated staphylococcal biofilms. Thus, other mechanisms must be involved in the antibiotic tolerance of these biofilms. The techniques described may prove useful in further examining the efficacy of advanced antibiotics designed to treat multidrugresistant biofilms.

Funding: Financial support for this work was provided by Theravance, Inc. (South San Francisco, CA). Telavancin was also supplied by Theravance, Inc.

Competing interests: None declared.

Ethical approval: Not required. 


\section{Appendix A. Supplementary data}

Supplementary data associated with this article can be found, in the online version, at http://dx.doi.org/10.1016/j.ijantimicag.2015. 05.022

\section{References}

[1] Scott II RD. The direct medical costs of healthcare-associated infections in U.S. hospitals and the benefits of prevention. Atlanta, GA: US Centers for Disease Control and Prevention; 2009. p. 1-13.

[2] Costerton JW, Stewart PS, Greenberg EP. Bacterial biofilms: a common cause of persistent infections. Science 1999;284:1318-22.

[3] Leadbetter MR, Adams SM, Bazzini B, Fatheree PR, Karr DE, Krause KM, et al. Hydrophobic vancomycin derivatives with improved ADME properties: discovery of telavancin (TD-6424). J Antibiot (Tokyo) 2004;57:326-36.

[4] Higgins DL, Chang R, Debabov DV, Leung J, Wu T, Krause KM, et al. Telavancin, a multifunctional lipoglycopeptide, disrupts both cell wall synthesis and cell membrane integrity in methicillin-resistant Staphylococcus aureus. Antimicrob Agents Chemother 2005;49:1127-34.

[5] Gander S, Kinnaird A, Finch R. Telavancin: in vitro activity against staphylococci in a biofilm model. J Antimicrob Chemother 2005;56:337-43.

[6] LaPlante KL, Mermel LA. In vitro activities of telavancin and vancomycin against biofilm-producing Staphylococcus aureus, S. epidermidis, and Enterococcus faecalis strains. Antimicrob Agents Chemother 2009;53:3166-9.
[7] Smith K, Gemmell CG, Lang S. Telavancin shows superior activity to vancomycin with multidrug-resistant Staphylococcus aureus in a range of in vitro biofilm models. Eur J Clin Microbiol Infect Dis 2013;32:1327-32.

[8] Dice B, Stoodley P, Buchinsky F, Metha N, Ehrlich GD, Hu FZ. Biofilm formation by ica-positive and ica-negative strains of Staphylococcus epidermidis in vitro. Biofouling 2009;25:367-75.

[9] Goeres DM, Hamilton MA, Beck NA, Buckingham-Meyer K, Hilyard JD, Loetterle LR, et al. A method for growing a biofilm under low shear at the air-liquid interface using the drip flow biofilm reactor. Nat Protoc 2009;4: 783-8.

[10] Buckingham-Meyer K, Goeres DM, Hamilton MA. Comparative evaluation of biofilm disinfectant efficacy tests. J Microbiol Methods 2007;70: 236-44.

[11] Hlady V, Buijs J, Jennissen HP. Methods for studying protein adsorption. Methods Enzymol 1999;309:402-29.

[12] Stewart PS, Davison WM, Steenbergen JN. Daptomycin rapidly penetrates a Staphylococcus epidermidis biofilm. Antimicrob Agents Chemothe 2009;53:3505-7.

[13] Rani SA, Pitts B, Stewart PS. Rapid diffusion of fluorescent tracers into Staphy lococcus epidermidis biofilms visualized by time lapse microscopy. Antimicrob Agents Chemother 2005;49:728-32.

[14] Theravance I. Package insert; 2013. http://www.vibativ.com/ [accessed 01.06.15].

[15] Saraf LJ, Wilson SE. Telavancin, a new lipoglycopeptide antimicrobial, in complicated skin and soft tissue infections. Infect Drug Resist 2011;4: 87-95. 